\section{RevistadePolítica Económica y Desarrollo Sostenible}

Centro Internacional de Política Económica para el Desarrollo Sostenible
Revista de Política Económica y Desarrollo Sostenible

EISSN: 2215-4167 • Vol. 6 (1) • Julio-Diciembre, 2020: 1-9

DOI: https://doi.org/10.15359/peds.6-1.2

URL: http://www.revistas.una.ac.cr/politicaeconomica

Revista electrónica semestral publicada por el Centro Internacional de Política Económica para el Desarrollo Sostenible

Universidad Nacional, Lagunilla, Heredia Apartado 2393-3000

\title{
Aspectos sobre el empleo y su remuneración previo y posterior a la crisis ${ }^{1}$
}

\section{Aspects of employment and remuneration before and after the crisis}

\author{
Zsuzsanna Rózsa Campos Tomcsányi ${ }^{2}$ \\ zsuzsanna.campos.tomcsanyi@est.una.ac.cr
}

Fecha de recibido: 31 de agosto de 2020. Fecha de corregido: 21 de octubre del 2020.

Fecha de publicación: 11 de noviembre del 2020

\section{Resumen}

El componente de trabajo, considerado en este artículo como el que las personas realizan para empresas, ha venido presentando una desmejora en sus condiciones de manera perceptible. Las condiciones de la población laboral y las explicaciones a las causas del desempleo han sido tema de discusión en diversos círculos de pensamiento histórico. A partir de la Gran Depresión se pone en tela de duda la noción de que el desempleo se da debido a que las personas no aceptan los trabajos disponibles o los pagos ofrecidos. Se expone brevemente la visión sobre el trabajo en modelos y tendencias presentes en algunas corrientes de pensamiento económico del siglo veinte y se discuten algunos aspectos que tienen impacto sobre las condiciones laborales imperantes en la economía.

Palabras clave: empleo, condiciones laborales, teoría económica, remuneración laboral, desempleo.

\begin{abstract}
The component of labor, understood in this article as the work performed by individuals for companies, has shown a perceptible decline in conditions. The conditions of the working population and explanations for the causes of unemployment have been topics of discussion in various circles of historical thought. From the Great Depression on, the notion that unemployment is caused by the decisions of individuals who reject jobs available or payments offered has been put to question. The view on labor models and trends present in some economic currents of thought in the twentieth century is briefly presented, and some aspects that impact on the prevailing labor conditions in the economy are discussed.
\end{abstract}

Keywords: employment, labor conditions, economic theory, labor remuneration, unemployment.

1 Esta editorial respondió a una convocatoria sobre el tema "Pensamiento y práctica de la Política Económica", en el marco del 25 aniversario del CINPE-UNA.

2 Licenciada en economía de la Universidad Nacional, y licenciada en Administración de Empresas en los énfasis de Contaduría, Finanzas y Mercadeo de la Universidad Estatal a Distancia. Actualmente se desempeña como economista en el Instituto Costarricense de Electricidad, donde labora desde 2003. Sus áreas de interés incluyen la economía laboral, economía de la innovación, desarrollo sostenible y estudios de equidad social y de género 
Revista de Política Económica y Desarrollo Sostenible • EISSN: 2215-4167

Vol. 6 (1) • Julio-Diciembre, 2020: 1-9

DOI: https://doi.org/10.15359/peds.6-1.2

Campos Tomcsány

URL: http://www.revistas.una.ac.cr/politicaeconomica

\begin{abstract}
"Anyone who is willing to work and is serious about it will certainly find a job. Only you must not go to the man who tells you this, for he has no job to offer and doesn't know anyone who knows of a vacancy. This is exactly the reason he gives you such generous advice, out of brotherly love, and to demonstrate how little he knows of the world".

-Citado por Paul Krugman (4 de agosto de 2020) de la novela The Treasure of Sierra Madre, de B. Traven, 1927.
\end{abstract}

\title{
Introducción
}

En su mayoría, los modelos económicos consideran dentro de sus funciones de producción el factor trabajo, definido como las horas laboradas necesarias para elaborar una unidad de producto. Los modelos sencillos incluyen como componentes generales de este tipo de funciones el trabajo y el capital. De ambos, quizás el que ha generado mayores conflictos (al menos en algunos círculos) es el primero y su remuneración. Sobre esta línea, el presente artículo se centra en las condiciones del empleo, entendido este como el que realiza una persona para una empresa o al servicio de otra, con el objeto de exponer algunos aspectos que afectan este tipo de trabajo remunerado.

Dentro del pensamiento neoclásico se considera el trabajo similar a cualquier otro factor productivo, con cantidades producidas y valoradas en una escala altamente dinámica que se ajusta perfectamente a los requerimientos de las empresas ${ }^{3}$. Como tal, la remuneración debe ser flexible tanto al alza como a la baja, al tiempo que su disponibilidad también es flexible, ya que en el mercado esta aumenta o disminuye dependiendo del precio al que se cotice y el valor que le concedan sus oferentes. En otras palabras, una empresa puede contratar las habilidades y capacidades de un trabajador, y con ello necesariamente también una porción de su tiempo ${ }^{4}$, convirtiendo ocio en horas laborales, si logra pagar la cantidad que el agente que las ofrece considera que vale esa hora adicional que no puede dedicar al descanso.

Podría decirse que este tipo de enfoque -aunque muy abstracto y cobijado por los supuestos y el objetivo del estudio de la economía como separado de las consideraciones sociales y de justicia- deshumaniza un componente profundamente humano.

3 Al respecto E.K. Hunt señala que Jean-Baptiste Say, conocido por la Ley de Say, infería que "no hay diferencia cualitativa, en la creación de utilidad, entre el esfuerzo del trabajo humano, por un lado, y la propiedad de capital, tierra y propiedad, por otro" (Hunt, 2015, p. 135) Traducción propia. Lo anterior coloca tanto a los trabajadores como a los propietarios de capital como agentes económicos, indistintos unos de otros. Los trabajadores ofrecen su capacidad laboral en el mercado de factores, donde los empresarios pueden adquirir o contratar tanto capital como trabajo. La capacidad de intercambiar el trabajo por capital, por ejemplo, puede reflejarse en las curvas de isocostos, donde un factor productivo puede reemplazarse por otro a una tasa marginal dada, para producir la misma cantidad del mismo producto (Varian, 2010). Ahora bien, si se considera tanto el capital como el trabajo como factores productivos, es posible hablar que uno puede reemplazar al otro. Empíricamente esto puede ser observado, por ejemplo, en empresas donde algunas funciones son automatizadas, pasando de la producción realizada por trabajadores a aquella realizada por máquinas.

4 De acuerdo con Hunt (2015), "The "freedom" of labor consists in the facts that the laborer can sell his or her power only for limited, contractually defined periods", lo que puede traducirse como "La "libertad" de trabajo consiste en el hecho de que el trabajador o trabajadora puede vender su poder por un período limitado, contractualmente definido". Lo anterior implica que la relación laboral puede interpretarse como una venta de un combo que incluye tanto la capacidad del trabajador como un espacio de tiempo determinado por medio del contrato laboral. 
Revista de Política Económica y Desarrollo Sostenible • EISSN: 2215-4167

Vol. 6 (1) • Julio-Diciembre, 2020: 1-9

DOI: https://doi.org/10.15359/peds.6-1.2

Campos Tomcsány

URL: http://www.revistas.una.ac.cr/politicaeconomica

Con la llegada de la Gran Depresión, esta línea de pensamiento económico presentó serias dificultades para explicar los fenómenos que se estaban dando, enfrentándose a la realidad de una población dispuesta a trabajar pero que no lograba obtener empleo. Efectivamente, no se trataba de oferentes que valoraban su tiempo de ocio por encima del precio por hora pagado por su trabajo. Las personas no estaban esperando una mejor oferta.

\section{Nuevos enfoques}

Nuevas teorías económicas surgieron para explicar estos fenómenos y ofrecer soluciones a un problema que ahogaba al mundo. Así es como entra en escena John Maynard Keynes y la propuesta, en su momento revolucionaria, que puso de cabeza las concepciones económicas en las cuales se fundamentaba esta disciplina. Su teoría es aplicada, luego criticada y posteriormente surgen nuevos seguidores, que son quienes traen a la mesa nuevos modelos económicos, como el de precios rígidos y el modelo interno-externo ${ }^{5}$.

El primer modelo mencionado, la de los precios rígidos, sostiene que los salarios, a diferencia de los precios de otros factores productivos, son rígidos hacia la baja, lo que implica que los mismos pueden aumentar, pero no disminuir (Froyen, 2015, pág. 385). La segunda teoría, por otro lado, indica que las remuneraciones son acordadas entre las empresas y los trabajadores contratados, dejando por fuera de este proceso a los desempleados (Froyen, 2015, pág. 386). Esta dinámica empuja el precio de las horas laborales por encima del fijado en el mercado, aprovechando en el proceso de negociación las ventajas que otorgan las organizaciones sindicales, y los costos de contratación y entrenamiento a que se enfrentan las empresas. Las condiciones pactadas tienen efecto tanto en las variaciones salariales a lo largo del proceso de empleo, como en la redacción de los contratos. Ejemplos conocidos de los logros obtenidos gracias a este poder de negociación de los trabajadores internos son los documentos de acuerdo entre patronos y empleados, conocidos con el nombre de convenciones colectivas.

A pesar de lo anterior, las economías siguen experimentando desempleo y variaciones en las condiciones de trabajo que parecen escaparse incluso de las normativas que han procurado cercarlas dentro de una serie de garantías sociales y laborales. Es así como se observan diferentes prácticas tendientes a altas rotaciones de empleados, o bien la minimización de la planilla y, por el contrario, realizar las actividades requeridas con profesionales independientes

o personal temporal. Este fenómeno es tratado también por Munck (2019), quien hace referencia a cómo las reformas estructurales aplicadas a las economías latinoamericanas en la década de los ochenta implicaron, entre otros temas, la flexibilización laboral. De acuerdo con este autor, es en esta etapa que la precarización laboral de América Latina se normaliza. Las medidas aplicadas erosionan las fortalezas de los trabajadores sobre los que se sustenta el modelo interno-externo. Si bien se mantienen de alguna manera las principales propuestas del

$5 \quad$ Las definiciones de estos modelos parten de lo expuesto por Froyen (2015), páginas 372-388. 
Revista de Política Económica y Desarrollo Sostenible • EISSN: 2215-4167

Vol. 6 (1) • Julio-Diciembre, 2020: 1-9

DOI: https://doi.org/10.15359/peds.6-1.2

Campos Tomcsány

URL: http://www.revistas.una.ac.cr/politicaeconomica

modelo de salarios rígidos a la baja, las prácticas logran efectivamente controlar el aumento de dichos salarios dentro de las empresas.

Estas estrategias de manejo del recurso humano, como es de esperarse, no son generalizadas a todos los puestos de trabajo ni a todas las empresas, sino que las mismas se concentran sobre las actividades y clases laborales más vulnerables, en cierta manera realizando un traslado y aplicación del modelo interno-externo con variaciones sutiles que permiten desgastarlo hasta el punto que resulta conveniente para clases particulares.

En 2017 Jay Shambaugh y Ryan Nunn analizaron el fenómeno de la relación entre el crecimiento de la economía y cómo se refleja este incremento para la mayoría de las personas. Para la medición del beneficio percibido por las personas, es necesario conocer, en primera instancia, cómo accede la población a sus ingresos.

Si bien es posible que las personas obtengan ingresos de diversas fuentes, el caso en particular de los autores se centra en los trabajadores que reciben un sueldo o salario por las horas laboradas para un tercero. A partir de esto se establece el supuesto de que los aumentos en la productividad de los trabajadores son necesarios para que estos experimenten incrementos en sus salarios. Sin embargo, ¿se da esto así?

Shambaugh y Nunn (2017) señalan que, aunque los salarios analizados en los Estados Unidos han mantenido un crecimiento ligeramente por encima de la tasa de inflación $(0,2 \%$ anual), la distribución de las ganancias por el crecimiento económico ha sido dispar a lo largo de los distintos grupos salariales. Así, quienes obtienen los beneficios del crecimiento de la economía son aquellos trabajadores que se encuentran en las escalas laborales más altas, mientras que los que se ubican en los niveles medios y bajos no perciben estas mejoras en sus ingresos.

Las razones de esto radican esencialmente en el poder de negociación que ostentan los trabajadores a lo largo de la estructura salarial existente, lo cual no es un fenómeno desconocido, y se ha normalizado en el ambiente laboral. Así, hoy en día se satanizan las asociaciones sindicales, pero se consideran normales los paracaídas de oro que pactan los gerentes dentro de sus contratos. El modelo de internos-externos de los neokeynesianos no ha desaparecido, sino que se ha transformado para convertir en internos a los ejecutivos, gerentes y asesores, y en externos al grupo compuesto por trabajadores de niveles medios y bajos junto a los desempleados.

Así, segmentos laborales importantes se van descalificando y la brecha salarial se ensancha cada vez más. A esto se agrega la erosión de las garantías laborales y los instrumentos con los cuales los trabajadores antes mencionados pueden ejercer presión para mejorar sus condiciones, lo cual continúa alimentando la disparidad. La erosión de las garantías laborales corresponde a diversas prácticas, como el no pago de horas extra, o bien su pago con el mismo monto del salario ordinario; el no aseguramiento del trabajador ante las entidades del seguro social, o bien su aseguramiento por montos inferiores. Si bien muchas de estas prácticas no son legales, no hay evidencia de una ejecución robusta de la ley, lo cual posibilita que estos procedimientos se mantengan y se continúen. 
Revista de Política Económica y Desarrollo Sostenible • EISSN: 2215-4167

Vol. 6 (1) • Julio-Diciembre, 2020: 1-9

DOI: https://doi.org/10.15359/peds.6-1.2

Campos Tomcsány

URL: http://www.revistas.una.ac.cr/politicaeconomica

En el caso de Costa Rica, si bien se han venido decretando aumentos salariales para los sectores público y privado de manera semestral hasta 2016 y de manera anual a partir de 2017, estos en ocasiones no han logrado superar el crecimiento de la inflación. Considerados los datos de 2016 a 2020, el aumento promedio en el salario real de los trabajadores es de un 0,12\%.

En el caso del sector público, y de acuerdo con la información del Ministerio de Trabajo y Seguridad Social (marzo, 2020), los salarios más bajos -que han recibido los aumentos más altos del sector- han perdido en promedio un $0,61 \%$ anual de su capacidad adquisitiva en el período comprendido entre 2015 y 2019. Los decrementos en los ingresos reales son más sensibles cuanto menor es el nivel salarial de inicio, a causa de las restricciones presupuestarias que impone la disminución en la capacidad adquisitiva.

Por otra parte, los niveles ejecutivos, gerenciales y similares cuentan con condiciones especiales que les permiten negociar sus remuneraciones cada cierto tiempo, no solo debido a sus cualificaciones particulares, sino también a los conocimientos especializados que adquieren durante su labor. La probabilidad de que un funcionario sea seducido por la competencia y pueda llevar consigo secretos empresariales es suficiente para que la entidad en la cual se desempeña esté dispuesta a pagar un premio para retenerlo.

Estas prácticas pueden pervertir el proceso de costos de la empresa, la cual, al momento de buscar hacer su estructura de costos más eficiente, puede decidir aplicar una serie de medidas de recorte a la cantidad de personal en los escalafones salariales bajos y medios, al tiempo que aumenta las cuotas laborales o metas de estos trabajadores, sin tocar los premios de los niveles considerados como estratégicos. Las prácticas empresariales de recortes cíclicos de personal, liquidaciones periódicas con recontrataciones solo de parte de la planilla anterior, podrían ser cada vez más frecuentes y llevarse a cabo justamente sobre los grupos de salarios más bajos.

En su artículo Shambaugh y Nunn mencionan también cómo la "caída del dinamismo" entre los trabajadores ha contribuido al estancamiento de los salarios en los niveles medios y bajos (30 de noviembre de 2017). Esto implica que la capacidad de negociación de la fuerza laboral disminuye cuando están menos dispuestos a trasladarse a otras áreas en busca de mejores oportunidades, o incluso a iniciar sus propias empresas.

En efecto, los montos de remuneraciones estancadas, y más aún aquellas cuya capacidad adquisitiva se reduce, impiden a los trabajadores poder ahorrar lo suficiente para poner en marcha un emprendimiento. A lo anterior se suman las condiciones cada vez más difíciles en los mercados financieros para obtener financiamiento adecuado para pequeñas y medianas empresas (pymes) y proyectos profesionales independientes, con lo cual se profundiza la posición de desventaja de este segmento de la población ante sus empleadores al momento de negociar mejoras salariales, por cuanto la amenaza de abandonar la empresa en caso de no obtener un aumento no está tan presente.

Los autores Shambaugh y Nunn (2017) hacen referencia a algunas opciones que podrían mejorar, desde la política económica y social, las condiciones de los trabajadores de los escalafones más bajos, como los aumentos salariales (ya instaurados en Costa Rica, con los 
Revista de Política Económica y Desarrollo Sostenible • EISSN: 2215-4167

Vol. 6 (1) • Julio-Diciembre, 2020: 1-9

DOI: https://doi.org/10.15359/peds.6-1.2

Campos Tomcsány

URL: http://www.revistas.una.ac.cr/politicaeconomica

resultados esbozados previamente) y el aumento del poder de negociación de los trabajadores, principalmente por medio de la intervención gubernamental en aspectos como la regulación de los contratos laborales y las cláusulas que puedan resultar restrictivas para los trabajadores.

Estas propuestas, sin embargo, ya cuentan con anticuerpos inoculados dentro de la corriente sanguínea de diversos sectores empresariales y laborales. Previo a la crisis sanitaria que actualmente vive el mundo, las organizaciones sindicales eran objeto de fuertes críticas y campañas de satanización, que desincentivaban a los trabajadores a unirse a las mismas. Por otra parte, la percepción de la población en este sentido puede ser que las denuncias de abusos interpuestas contra patronos no prosperan, habida cuenta de que las prácticas laborales previamente mencionadas continúan dándose y propagándose, con lo cual se continúa erosionando la fuerza de los trabajadores, particularmente de aquellos con menor capacidad para negociar sus condiciones.

Las prácticas salariales como las anualidades, carrera profesional, carrera académica y ajuste del percentil también han venido criticándose bajo la premisa que se trata de privilegios y dádivas asociadas a retribuciones por ineficiencia o vagancia. Independientemente del aporte con que los trabajadores hayan contribuido a la economía en su conjunto, los esquemas de distribución equitativos se enfrentan a fuertes campañas de desprestigio mientras que las remuneraciones y premios de los estratos altos se justifican, incluso cuando los logros sobre los cuales se apoyan no serían posibles sin el esfuerzo de aquellos empleados a quienes se les niega su participación en los beneficios, tal y como lo ilustran Shambaugh y Nunn (2017).

Las prácticas laborales cada vez más agresivas, donde la reducción de costos muchas veces se realiza en detrimento de los trabajadores o con prácticas ilegales tales como evasión fiscal, evasión en los pagos del seguro social, el no pago de cesantías, así como diversas prácticas de elusión fiscal, ha contribuido a la cristalización de condiciones cada vez más precarias para los escalones medios y bajos de la estructura salarial.

En esta situación se enfrenta el mundo a una pandemia mundial que ataca no solo la salud, sino la economía también, y donde se ponen en evidencia las prioridades y las necesidades de los diferentes segmentos. Las empresas esperan recibir asistencia de los gobiernos, mientras en distintos medios se consideran como una solución incorrecta los fondos girados directamente a apoyar a las personas que han perdido su empleo, por diversos motivos. Así como se observa en Costa Rica, se puede apreciar en otros países, donde cala la idea de que las ayudas para los desempleados solo promueven el ocio. Al respecto, Paul Krugman, economista norteamericano, se ha referido a las fórmulas ya desacreditas con que se intenta desprestigiar las ayudas por desempleo como "conceptos zombies" o “teorías zombie" (Krugman, 2020).

Krugman ha escrito diversos artículos al respecto, señalando recientemente que la ayuda de desempleo no solo no incentiva la indolencia, sino incluso si un trabajador aceptara trabajar por menos, cuando lo hagan todos los demás desempleados -en un medio donde las medidas sanitarias reducen las posibilidades laborales- nuevamente se volverá al escenario donde son más 
Revista de Política Económica y Desarrollo Sostenible • EISSN: 2215-4167

Vol. 6 (1) • Julio-Diciembre, 2020: 1-9

DOI: https://doi.org/10.15359/peds.6-1.2

Campos Tomcsány

URL: http://www.revistas.una.ac.cr/politicaeconomica

los desempleados que los puestos ofrecidos. ¿Se podrá continuar diciendo entonces que quien no encuentra trabajo es por su propio deseo de ser mantenido por el Estado? (Krugman, 2020).

El punto que convenientemente queda por fuera es ¿cuán baja debe ser la remuneración de ese trabajador si una ayuda social es superior al sueldo que puede recibir en una empresa? Lo que lleva a la pregunta de si la oposición a esta ayuda es porque, justamente, le otorga una ínfima oportunidad al trabajador de negociar una mejora en su salario.

Stiglitz (2020) considera un aspecto diferente de la economía y la crisis, observando la necesidad de lograr una reactivación económica efectiva en medio de la pandemia, y defiende la importancia de impulsar esta reactivación por medio de los componentes de consumo e inversión del producto interno bruto (PIB); además discute cómo las políticas implementadas no han surtido el efecto deseado. Por el contrario, Stiglitz menciona cómo las empresas receptoras de estos fondos prefieren ahorrarlos dada la incertidumbre que enfrentan en los mercados en que operan, con lo cual se pierde el objetivo de la política.

Los recursos provenientes de estos planes de reactivación no se inyectan de vuelta a la economía; ante el panorama existente es posible que pase un tiempo considerable antes de que las empresas puedan volver a funcionar con normalidad. Esto lleva nuevamente a valorar las prácticas mencionadas de previo, donde los recortes se aplican desproporcionalmente a los trabajadores de rangos medios y bajos, protegiendo a los de niveles más altos.

Si bien el artículo de Stiglitz pone énfasis en la necesidad de incentivar el consumo en momentos de crisis para poner nuevamente en marcha el mercado, la pregunta vuelve al punto de partida: ¿cuáles son los sectores de los que se espera este consumo aumentado? Una pregunta cuya respuesta necesariamente se verá reflejada en la formulación de políticas.

La presión hacia el consumo de las personas en el mercado, en un ambiente previo a la pandemia, no ha aminorado incluso para los sectores medios y bajos, los cuales usualmente recurren a créditos hipotecarios y personales con tasas muchas veces punitivas. En un ambiente de crisis sanitaria, junto con la crisis económica y laboral que trae consigo una contracción sustancial del consumo de estos sectores, particularmente cuando se flexibilizan las políticas laborales y las personas pierden sus ingresos, la disparidad ya presente en las estructuras salariales resulta aún más marcada.

Así, si bien se aboga en diversos sectores por la flexibilización salarial, ya no solo en el marco de la crisis sanitaria, sino como un cambio a operarse de manera progresiva y permanente en el contexto laboral, invalidando el modelo de precios fijos para este sector, se fortalece el modelo interno-externo, donde quienes no disfrutaban de los beneficios del crecimiento económico ahora cargan con la parte leonina de la contracción. La pregunta que queda es: ¿puede recuperarse la economía y lograr mayor equidad con las propuestas de Shambaugh y Nunn, apostando no solo al aumento de salarios mínimos, sino también al fortalecimiento del poder negociador de la clase trabajadora? ¿O se requiere tomar una página de las propuestas de Stiglitz, dirigidas no solo al fortalecimiento de las empresas, sino también al apoyo del consumo 
Revista de Política Económica y Desarrollo Sostenible • EISSN: 2215-4167

Vol. 6 (1) • Julio-Diciembre, 2020: 1-9

DOI: https://doi.org/10.15359/peds.6-1.2

Campos Tomcsány

URL: http://www.revistas.una.ac.cr/politicaeconomica

por parte de las personas mediante inyecciones de fondos provenientes de ayudas del Estado cuando las condiciones lo ameriten? Porque no solo en la crisis son necesarias para muchos las ayudas, si es que su capacidad de negociación no es suficiente para que su productividad sola les permita acceder a los beneficios de los que gozan estratos más altos de la economía.

\section{Conclusiones}

Una política formulada en estos tiempos debe no solo dirigirse a la situación de la crisis sanitaria en que se encuentra inmersa la economía, sino que debe procurar también la proyección al futuro. El apoyo al sector productivo no debe darse en detrimento de la fuerza laboral, por lo que es necesario que los incentivos se acompañen con un robustecimiento de las condiciones en que se desarrolla el empleo, así como la creación de programas de apoyo sostenibles para desempleados y la población en condiciones de vulnerabilidad. Gran parte de la estructura para el fortalecimiento de las condiciones laborales existe, pero requiere de su aplicación adecuada, como es el caso de las garantías laborales.

Un mayor esfuerzo en la lucha contra la evasión y la elusión, mayor seguimiento de las condiciones laborales en las distintas empresas son acciones que permiten aprovechar el utillaje ya existente. Sobre esto se pueden construir propuestas adicionales, como esfuerzos para facilitar la formalización de trabajadores informales, de modo que sea posible extenderles a ellos también las ventajas propias de los trabajadores en la formalidad. El desarrollo de programas que fortalezcan la posición de los trabajadores de escalafones salariales inferiores, por medio de opciones de capacitación a los cuales puedan tener acceso fuera de sus horarios laborales, así como la promoción de bolsas de empleo, mayores facilidades para el acceso al crédito para pequeños emprendimientos que promueva el dinamismo al que hacen referencia Shambaugh y Nunn (2017).

Quizás el primer paso es desterrar las ideas enconadas sobre los desempleados y los trabajadores de las escalas bajas y medias -esas posturas que Krugman llama "ideas zombies"-, reconocer su importancia en la economía, para proceder posteriormente a los cambios necesarios en el resto de la estructura, incluida la educación y capacitación para reincorporarse o mantenerse en los nuevos mercados laborales ajustados a la postcrisis, que dicho sea de paso están cambiando enormemente. Pero mientras las fuerzas económicas se muevan a favor de quienes acaparan los beneficios, y promuevan las medidas y los mensajes dirigidos a socavar las oportunidades y el acceso del resto, cualquier idea y cualquier propuesta terminará ahogada y abandonada antes de siquiera considerarlas.

\section{Referencias}

Froyen, R. (1995). Macroeconomía. Teoría y Políticas. Bogotá: McGraw-Hill Interamericana, S.A. 
Revista de Política Económica y Desarrollo Sostenible • EISSN: 2215-4167

Vol. 6 (1) • Julio-Diciembre, 2020: 1-9

DOI: https://doi.org/10.15359/peds.6-1.2

Campos Tomcsány

URL: http://www.revistas.una.ac.cr/politicaeconomica

Hunt, E. K. (2015). History of Economic Thought, a Critical Perspective. Belmont, California: Wadsworth Publishing Company, Inc.

Krugman, P. (28 de julio de 2020). Zombies are stalking America's unemployed. En The New York Times. Columna de opinión.

Krugman, P. (4 de agosto de 2020). The legend of the lazy unemployed. En The New York Times. Columna de opinión.

Ministerio de Trabajo y Seguridad Social (marzo, 2020). Anuario Estadístico 2019. Dirección General de Planificación del Trabajo, Observatorio del Mercado Laboral. San José, Costa Rica: Autor.

Munck, R. (2019). The Labor Question and Dependent Capitalism: The Case of Latin America. En The Social Question in the Twenty-First Century. University of California Press.

Shambaugh, J. y Nunn, R. (30 de noviembre de 2017). ¿Por qué crece la economía pero no los salarios? El caso de Estados Unidos. En Harward Business Review en Español. Recuperado de https://www.hbr.es/econom/916/por-qu-la-econom-pero-no-los-salarios-el-caso-deestados-unidos

Stiglitz, J. (8 de junio de 2020). Un estímulo que funcione. En Project Syndicate. 